Voix et Images

\title{
Est-il chose plus belle qu'une orange? Rencontre avec Rina Lasnier
}

\section{Joseph Bonenfant et Richard Giguère}

Volume 4, numéro 1, septembre 1978

Rina Lasnier

URI : https://id.erudit.org/iderudit/200133ar

DOI : https://doi.org/10.7202/200133ar

Aller au sommaire du numéro

Éditeur(s)

Les Presses de l'Université du Québec

ISSN

0318-9201 (imprimé)

1705-933X (numérique)

Découvrir la revue

Citer ce document

Bonenfant, J. \& Giguère, R. (1978). Est-il chose plus belle qu'une orange?

Rencontre avec Rina Lasnier. Voix et Images, 4(1), 3-32.

https://doi.org/10.7202/200133ar d'utilisation que vous pouvez consulter en ligne.

https://apropos.erudit.org/fr/usagers/politique-dutilisation/ 


\section{Est-il chose plus belle qu'une orange? Rencontre avec Rina Lasnier ${ }^{1}$}

\section{Les années de formation}

J. B. Comment avez-vous découvert la poésie?

R. L. Comme tout écolier et enfant, dans mes manuels.

Cependant, je suis tentée de vous raconter une belle histoire vraie. Je ne la raconterais pas si ce fait n'avait été, plus tard, authentifié par ma mère.

J'avais environ deux ans, et ma mère souffrante avait dû me confier à ma grand-mère maternelle pour plusieurs semaines.

Lorsque mes grands-parents me ramenèrent à ma mère, je voyageais dans les bras de ma grand-mère, en voiture-traîneau puisque c'était l'hiver.

Soudain, je vis pour la première fois, je pris une parfaite conscience de deux choses : d'abord de la tombée silencieuse de la neige... prodige qui m'éveilla complètement de l'inconscience et me révéla en même temps, la douceur du visage de ma grand-mère à demi voilée par cette neige dense et magique.

Lorsque ma mère voulut me saisir pour me déballer de mes enveloppements de voyage, je criai et protestai, encore sous le coup du prodige, ne reconnaissant plus ma mère, bouleversée de mon oubli. Puis, ce fut à nouveau la nuit de l'enfance nuageuse et qui ne retient dans sa mémoire fragile que quelques éclairs de bonté ou de beauté, et parfois de douleur. Plus tard, il $y$ eut les bibliothèques. II y avait aussi un libraire-papetier, comme il en existe dans toutes les petites villes. $\mathrm{Ne}$ connaissant même pas l'existence de l'Index, ou feignant de l'ignorer, ce cher libraire passait n'importe quoi à des adolescentes de 12, 13 et 14 ans. Grâce à cette largeur de vue, c'est à cet âge que j'ai lu mon premier roman scabreux. II va de soi que je tirais de ce fouillis les "trésors" et anthologies de la poésie. Je devais être la seule à m'intéresser à la poésie et je n'en parlais jamais. C'était un secret, non pas honteux mais bienheureux. 
Songez qu'à cette époque, dès l'âge de douze ans, nous écrivions toutes sans fautes ou presque. Cependant, c'est à mes lectures de la poésie que je dois mon premier prix de français. C'était la coutume, pour l'examen de français, que chaque classe reçoive la Mère Provinciale pour l'épreuve de la dictée. Or, le mot rare «nautonier» se trouvait dans cette dictée. Je fus la seule à l'orthographier correctement. La Mère Provinciale, intriguée, me demanda où j'avais lu ce mot. Je répondis : "dans Victor Hugo"; et je fus décorée d'un beau ruban rouge pour cette fréquentation de la poésie et cet enrichissement du vocabulaire.

J. B. Quelles ont été vos lectures?

R. L. Tout ce que je pouvais emprunter à la maigre bibliothèque de la paroisse, à mes jeunes amies. Naturellement, la poésie restait pour moi le haut lieu d'une sorte de connaissance et d'émoi que je ne pouvais ni nommer ni partager. Je découvris un jour, dans notre mince bibliothèque de classe, une anthologie de poèmes d'amour hindous. Je m'en emparai, malgré la défense formelle de toucher à ce rayon réservé. Ma désobéissance fut dûment punie, mais je restais sans remords.

J. B. C'était donc à l'Index?

R. L. Non, mais je me demande encore aujourd'hui comment ces poèmes étaient parvenus jusqu'à notre pauvreté d'écolières curieuses de la vie... et de l'amour.

Cette lecture interdite comme la pomme à Ève m'éveilla à un autre langage de la poésie. J'avais quand même quinze ans à cette époque. Et pourtant, je crois que déjà je m'initiais à cette poésie à la fois charnelle et spirituelle qui marquera si fort la mienne.

R. G. Vous étiez la seule, dites-vous, à lire de la poésie. Était-ce un goût de famille?

R. L. Je ne crois pas... à moins que l'on retienne que la musique, dans notre famille, a tenu une large place. Mon père jouait du violon, par oreille, composait des "gigues " la nuit; ma sceur aînée avait tous les diplômes de l'époque en piano, et chantait fort agréablement.

J. B. Comment êtes-vous revenue à la poésie orientale?

R. L. Par la route habituelle de la tentation... je veux dire des visites au rayon des livres de la maison Morgan qui, à cette époque, offrait un choix exotique assez complet. Je préparais alors mon baccalauréat, raison de plus pour m'évader de la poésie française, plus intellectuelle, vers la poésie orientale, vers les quatrains des ruba'iyyat que j'ai lus et relus. Puis ce furent les poètes soufis, 'Attār, Rūmí, Saadi aux roses. Que me convenaient ces sages, ces mystiques passant si simplement et si profondément du naturel au surnaturel, et inversement, 
ne laissant rien de la faim des hommes emmêlée à la faim de Dieu, comme le corps mêlé à l'âme! Poètes beaucoup plus près de la Bible, de l'Ancien Testament que tous les poètes que j'avais fréquentés dans les livres français.

Étrange, mais c'est dans la poésie anglaise que je retrouvai cette intimité, cette chaleur de l'humain se frayant un chemin jusqu'à la mystique ; songez à Francis Thompson, Hopkins...

R. G. C'est drôle, vous n'avez pas commencé par notre poésie...

R. L. C'était plutôt normal de ne pas connaître la poésie canadiennefrançaise, car personne ne l'enseignait, ne nous la faisait connaître.

R.G. DesRochers disait la même chose.

J.B. Vous, vous découvrez la poésie anglaise par des études en Angleterre. Qu'est-ce qui vous amène en Angleterre?

R. G. Le désir de mes parents de nous faire apprendre l'anglais. L'Ontario nous était comme interdit à cause de l'hostilité larvée jusque dans les institutions catholiques. Les États-Unis ne valaient guère mieux parce que les Canadiennes françaises des couvents américains faisaient bloc et mettaient beaucoup de temps à maîtriser l'américain.

Puis, il faut le dire, nous avions une parente en Angleterre, une religieuse qui nous invitait fortement à étudier à Palace Gate, institution privée taillée à même le domaine du palais épiscopal protestant. Nous y fûmes très heureuses, ma soeur et moi. Régime de vie, d'étude totalement différent du nôtre. Plus de soins, d'exigences pour la formation et l'éducation parfaite que pour les diplômes et les compétences. Et c'est par un ballet que les élèves accueillaient la venue de l'évêque; j'ai donc dansé sur l'herbe pour saluer un bel Anglais mitré... Sainte audace si on la compare à nos profondes révérences et à nos gants noirs du Québec, dans une même circonstance... Sans compter que nous apprenions l'anglais comme sans effort et avec beaucoup de lectures libres... et de promenades interminables dans la campagne romantique et pastorale du Devonshire.

R. G. Vous aviez quel âge?

R. L. Dix-sept ans.

J. B. Qu'est-ce que vous avez lu?

R. L. Surtout les classiques. On nous faisait même jouer des extraits de Shakespeare; ce devait être pour distraire les religieuses de la maison et les douze pensionnaires, étrangères que nous étions toutes.

J. B. Votre séjour correspondait à une ouverture sur le monde de l'esprit et de la culture. 
R. L. Sur un monde de l'esprit et de la culture... anglais. Très anglais puisque Palace Gate avait d'abord été une institution privée réservée à la seule noblesse. Dès que la direction commença à accepter des écolières non titrées, des filles d'ambassadeurs, de médecins, etc., la noblesse se retira. Lorsque nous y fûmes, ma sœur et moi, les pensionnaires représentaient cinq ou six nationalités.

R. G. Votre mère lisait plus que votre père?

R. L. Oui, mon père n'a jamais lu, mais demandait à ma mère la lecture des journaux ou la communication de ses lectures à elle, surtout ce qui touchait la politique. Mon père venait d'une famille pauvre et nombreuse et perdait son père dès l'âge de deux ans environ. La mère, restée veuve, devait arracher sa vie d'une petite terre pauvre et les enfants ne pouvaient flâner ni à l'école ni à la maison. C'est pour cette raison, je pense, que mon père avait pris la campagne et l'agriculture en grippe et se lança dans les affaires dès son mariage avec ma mère. II acheta un hôtel dans un petit village (celui qui vit naître le Frère André) et réussit dès le départ, tout en se chicanant avec son curé qui le citait en chaire et dénonçait les ventes d'alcool ou de bière aux terrassiers de la paroisse, presque tous Polonais.

J. B. Si l'on poursuivait avec la découverte de la littérature anglaise?

R. L. Tout en préparant mon baccalauréat au collège MargueriteBourgeoys, je suivais les cours de littérature anglaise à l'Université de Montréal, de même que les cours de littérature française. J'élargissais, j'approfondissais ce que j'avais commencé à Exeter (Angleterre). Je découvrais aussi la poésie américaine. Je ne sais pourquoi, peutêtre à cause du dépaysement d'une langue étrangère, j'ai toujours eu l'impression que la poésie anglaise restait plus près de ses sources, celles de la tradition et celles du poète. Elle fait moins de bruit intellectuel ; elle va plus directement à la concision, au rêve, à l'indéfini.

J'aimerais disposer de plus de temps et traduire des poèmes anglais, comme j'ai tenté de traduire quelques poèmes de A. J. M. Smith, et surtout, comme j'ai été traduite par madame G. Downes qui me trahit magnifiquement, faisant d'un poème une légende... Ainsi, il peut lui arriver, pour transposer, de commencer la traduction d'un poème par le dernier vers, remontant le torrent au lieu de s'y livrer aveuglément.

J. B. D'autres lectures?

R. L. Certes, écrivains allemands, sud-américains. J'aimerais connaître l'allemand pour lire les poètes dans le texte. Il me semble, est-ce une illusion ou est-ce le souvenir du romantisme allemand vécu en profondeur jusqu'à l'excès démentiel et jusqu'à la philosophie durement idéaliste, il me semble que la poésie allemande a davantage le sens de l'exil du poète comme de son enchantement parfois mortel. Songez à Hölderlin, Novalis, Rilke que j'ai lus en entier. A propos de 
Rilke, j'ai découvert avec sympathie que les débuts de ce grand poète avaient été assez pénibles. Serait-il parvenu si tôt à cette maîtrise de son dire sans l'influence de Lou Andréas-Salomé ?

J. B. Vous lisez Hopkins en anglais?

R. L. Bien sûr. Tout en admirant l'extraordinaire, j'allais dire la miraculeuse traduction de P. Leiris. Aussi exceptionnelle que la traduction des Sonnets de Louise Labé par Frederic Prokosch.

J.B. Et vos auteurs préférés?

R. L. Disons plutôt, tous les beaux poèmes de la poésie... Bien sûr, les plus grands restent à leur rang, même avec leurs faiblesses. Mais il arrive qu'un poète, inconnu ou méconnu, nous touche plus que les plus brillants... Ce sont des astres séparés... Je nomme ici, à titre d'exemple, René Ménard de Paris. Qui le connaît? Et pourtant les poèmes de Feuillage forment un tout magnifique. Mélange heureux de l'onirique et du sculptural, du secret et de l'ouvert.

J. B. II y a d'autres noms?

R. L. A quoi bon les dire, cela ressemblerait à une litanie de la poésie moderne. J'ajouterai cependant que je suis un peu étonnée qu'ici on connaisse et pratique peu Segalen, précurseur sans doute de SaintJohn Perse. Ou encore qu'on dédaigne Lanza del Vasto au profit de ses écrits contre la violence...

J. B. Et Valéry?

R. L. Beau diamant stérile...

J. B. Tout de même sensuel...

R. L. Certes, mais une 'sensualité brûlée par l'intelligence comme le désert par son soleil et ses mirages. Prose superbe, la plus parfaite peut-être de son époque.

J. B. Claudel, sans doute, est des vôtres?

R. L. Certes et au tout premier rang. Comment ne pas acquiescer à cette poésie, à ce théâtre, heureusement lyriques, recourbant l'humain jusqu'à la transcendance, l'alpha rejoignant l'oméga; et comment ne pas reconnaître en lui l'Adam libre dans les jardins de la Nature, le témoin d'un amour appelé à la plénitude de l'amour.

R. G. Connaissez-vous les surréalistes?

R. L. Je les connais pour les avoir traversés sans pouvoir y séjourner. On entre en cette poésie pour l'artifice et l'évasion, on en sort par le désespoir et la fermeture. Nous le savons encore mieux aujourd'hui, la pure spontanéité dissout presque toujours la poésie et aussi la conscience. Pensez à la présomption, parfois achevée en suicide, 
d'arracher à une forme de poésie un absolu, une idole, ou du moins une révolution du langage.

Retenons cependant que le surréalisme a provoqué une certaine libération de la notion même de poésie, mais l'indiscipline fondamentale du mouvement a fait avorter ce relais rigoureux de l'expérience poétique.

J. B. Je ne doute pas que Marie Noël soit parmi vos lectures?

R. L. Oui, prose et poésie. Cette femme de passion porte encore le masque de la dévote qui chante son âme de silence... Et pourtant ce grand poète n'a cessé de cheminer entre l'enthousiasme et le désespoir, le pathétique et l'ironie, la lucidité et la contradiction, la bénédiction et la malédiction. Sa poésie, parfaitement maîtrisée, accuse la plus grande liberté de ton, de rythme et de couleurs.

On sait que Montherlant ne reconnaissait que deux génies en France, Colette et Marie Noël. On sait aussi que Marie Noël admirait sa consoeur, comme elle bourguignonne. Si Marie Noël avait eu la vie épicée et scanleuse de Colette, sa gloire parisienne eut été assurée...

J. B. A quelles relectures revenez-vous le plus volontiers?

R. L. Cela dépend tellement de l'humeur, de la saison même, de ce que j'écris moi-même. Un seul exemple. J'en suis à une série de poèmes sur l'hiver. Je me souviens du poète suédois Erik Lindergren et j'ouvre le Sacre de l'hiver. J'y trouve ou retrouve une sorte de catharsis de l'hiver; et cela devient une musique qui provoque la mienne. Mais existent aussi des besoins complètement désintéressés.

J. B. Les autres lectures importantes?

R. L. Certaines sont en rapport avec mon travail. Ainsi avant d'écrire la Malemer, j'avais lu The Sea Around Us de Ruth Carson et quelques autres ouvrages sur les océans.

Jamais je ne me borne à un seul genre d'ouvrages pour une soirée de lectures. Je m'intéresse à tout... philosophie, essais littéraires et scientifiques, musique, peinture, etc.

J.B. Je suppose que vous faites aussi des lectures en théologie?

R. L. Vous me faites sourire. Je ne suis pas théologienne... Je lis donc plutôt des ouvrages de spiritualité.

J. B. Et l'exégèse biblique?

R. L. La Bible, oui, pas trop d'exégèse. Mes voies vont plutôt vers la contemplation que vers la science. J'aime mieux entrevoir Dieu dans Sa Parole que de décortiquer les hypothèses, souvent douteuses des exégètes. 
L'idéal, pour moi, c'est de tomber sur un théologien contemplatif. Je songe en ce moment au cardinal Garrone; ou encore à Yves Raguin, spécialiste des religions orientales. Comme ces religions ont en ce moment la faveur de plusieurs pays occidentaux, dont le Québec, nous n'avons plus droit d'ignorer la mystique chinoise, hindoue. La simple foi du charbonnier est périlleuse; la preuve en est que le petit peuple mord plus vite aux erreurs religieuses qui foisonnent en ce moment que le clergé de vive foi et les intellectuels de bonne foi.

Écoutez un brave type, néophyte de sa nouvelle foi, discuter de tout ce qu'on lui a vaguement appris et vous aurez envie de pleurer ou de rire. II semble avoir tout lu et tout digéré de la Bible ou des mystiques de l'Inde, alors qu'il n'a jamais lu le Nouveau Testament en entier ni surtout, l'Histoire de l'Église.

\section{L'entrée dans l'écriture}

R. G. Comment avez-vous été amenée à l'écriture?

R. L. Presque par inadvertance et surtout par repliement forcé sur moimême, au moment d'une maladie plus lente que grave. Dans ma famille on est porté vers la médecine. Je me destinais donc à cette discipline en faisant mon baccalauréat. J'ai une sœur infirmière, maintenant aux recherches à l'Institut Armand-Frappier. Mon frère, homme d'affaires, se serait consacré à la médecine si mon père l'avait laissé libre... de ne pas le remplacer à la tête de ses affaires.

Je vous ai dit que j'avais été mise au repos complet, pour mononucléose, immédiatement après mes études préparatoires. Que faire? Patienter, passer à travers toutes les défenses de lectures, d'études et se bâtir une existence acceptable. Prendre tous les divertissements et dérivatifs utiles ou agréables : fréquentation des amis et amies invités à la maison, séjour dans les Laurentides. Pas de sortie le soir, long repos le matin. Et ce soir et ce matin devinrent pour moi un grand et profond moyen d'intériorisation. Étrange, mais je m'habituais peu à peu à beaucoup de silence sans ennui aucun, au contraire, comme un plongeur sous-marin, je découvrais non seulement l'imaginaire mais les réserves de l'âme, puits pas assez exploré... voix pas encore assez écoutées et reconnues.

Comme je vivais beaucoup à l'extérieur, la nature me devint une compagne de prédilection et de sagesse indéfinie.

J.B. Quel fut l'événement le plus marquant de cette réclusion?

R. L. Pendant cette réclusion ouverte de dix-huit à vingt-quatre mois, bien installée au lit ou dans un fauteuil de jardin, je me mis tout simplement à écrire... pour passer le temps et passer du temps au sur-temps de la création. 


\section{J. B. Qu'avez-vous écrit?}

R. L. Féerie indienne, sorte de jeu ou d'oratorio sur Kateri Tekakwitha. $\mathrm{J}$ 'avais toujours admiré cette jeune Iroquoise de mon Richelieu qui avait tout quitté, comme Abraham, pays, famille, sécurité, pour rejoindre Dieu à Oka et sur le Saint-Laurent.

R. G. Vous nous avez dit antérieurement que vous aviez commencé par le journalisme?

R. L. Disons qu'après Féerie indienne, je repris ma vie normale. Avant de m'orienter vers un travail ou une activité nouvelle (le médecin ayant interdit les études prolongées de l'université), j'acceptai la responsabilité d'une page féminine au nouveau journal de Saint-Jean, le Richelieu, dont le rédacteur en chef était l'un de mes amis, Paul L'Écuyer.

J'ai tenu cette page hebdomadaire pendant près de cinq années sans salaire...

J. B. Que contenait cette page, ne nuisait-elle pas à votre écriture personnelle?

R. L. Cet exercice suivi d'écriture concise et rapide ne pouvait que me discipliner et m'habituer à la diversité. Comme j'avais commencé par bannir les recettes de cuisine de cette page où la femme me paraissait traitée en mineure ou en simple ménagère, vous devinez bien que la littérature, l'art, et même, l'astrologie eurent leur place. Je tâtai d'un courrier-étrange, mais personne ne mordit à l'hameçon. Et personne non plus, parmi ces dames ne consentit à collaborer. Ouf! ce que j'ai dû en remplir des colonnes et des colonnes, citant des extraits de poésie ou de proses. A telle enseigne que le journal eut à acquitter une amende pour mes largesses d'auteurs français... Mon bénévolat me dispensait heureusement de cette amende.

J. B. Le mot journal me force presque à vous demander si vous teniez un journal personnel?

R. L. Oui. J'ai commencé à l'âge de treize ans à me croire adulte et cela par une réaction psychologique contre la solitude. Un jour, ma sœur, à peine mon aînée, et que je suivais comme son ombre, parce que ma mère nous conseillait de rester ensemble et qu'au couvent on m'avait fait sauter une année afin que je puisse avoir les secours de ma sœur, donc un jour où cette consigne de surveillance avait exaspéré son obéissance, je vis avec stupeur la complaisance fraternelle se changer en colère et je m'entendis dire : «J'en ai assez de ta compagnie, trouvetoi des amies. " Et ma sœur disparut avec son amie à elle, à la rencontre de deux nouveaux amis.

Ce fut un tournant nécessaire mais douloureux. Je me sentis aussi seule qu'une orpheline au milieu d'une ville inconnue... Et je me réfugiai au journal intime, puis me découvris des amies à moi... 
J. B. Avez-vous eu, avant Féerie indienne, des indices de votre goût pour la poésie?

R. L. Mes lectures d'enfant pouvaient être une indication. Personnellement, la poésie me paraissait trop haute, trop difficile, trop privilégiée pour que je puisse jamais rêver d'en écrire.

Cependant, alors que j'étais en philosophie, au collège MargueriteBourgeoys, il y eut un concours intercollégial et je fus désignée pour traduire le poème anglais «Treès". Ce que je fis... Puis un jour, mon professeur de physique me demande à son cabinet et me dit, mi-rieuse mi-vexée: «Rina Lasnier, vous venez d'obtenir le premier prix intercollégial... mais hors concours. Vous n'avez pas traduit le poème, vous l'avez interprété..." ". Je reçus le ruban rouge, mais pas la somme de cent dollars, restée aux mains du traducteur fidèle...

J. B. Vous aviez donc déjà une grille personnelle d'écriture?

R. L. Probablement... j'ignore... Je sentais d'évidence que traduire littéralement détruisait le poème. Je voulus donc lui laisser sa plénitude de beauté et de mystère... et je passai à côté de la récompense mais non à côté de la poésie, peut-être.

J. B. On se demande comment vous avez alors gagné votre vie?

R. L. Je m'y préparai par un cours de bibliothéconomie. Avec ce diplôme, j'aurais eu une situation à Saint-Jean puisque l'évêque voulait me retenir pour le poste de directrice. Mes études terminées, je me présente à mon évêque qui m'apprend qu'il s'agit encore de bénévolat puisque «mon père peut me faire vivre "...

Mon père met son veto... et je rentre à la maison avec un diplôme dont je ne me suis jamais servi, sauf pour faire mes propres fiches d'écrivain.

J. B. Vous n'avez donc jamais travaillé à l'extérieur?

R. L. Non, comme journaliste, je faisais tout mon travail à la maison. Ensuite, j'ai accepté différentes collaborations, mais toujours en travaillant chez moi.

J. B. Pour un jeune écrivain, la vie princière quoi?

R. L. II ne faudrait pas oublier les difficultés inhérentes à une situation dépendante, ni une certaine fragilité de migraines et d'insomnie.

Je dois quand même avouer qu'une certaine vie mondaine, surtout pendant l'été, a bien rempli et coloré ma jeunesse. Nous pratiquions alors les sports nautiques et profitions largement du Richelieu : natation, canotage, excursions, puis tennis, golf. Le soir, nous dansions beaucoup, surtout avec les cadets-officiers, en stage à l'École militaire. Tout se passait très bien car à cette époque les clubs privés ne toléraient aucun alcool, pas même la bière... De plus, l'aumônier des jeunes officiers nous invitait souvent à sa tente, à l'heure du thé... 
J. B. Et la vie intérieure découverte en temps d'immobilisation?

R. L. J'aimais trop le silence et la lecture pour ne pas me réserver des temps de solitude et d'interrogation nostalgique ou rêveuse. Puis, j'avais un grand ami, étudiant en droit et déjà lettré, et aussi poète, qui m'écrivait pour me gronder, prévenir fermement contre la futilité et le gaspillage des vacances, de tout plaisir.

Ce correspondant sérieux étant presque fiancé à une compagne de collège, notre amitié restait parfaitement belle et désintéressée et toute remplie de poésie.

R. G. A ce point de vue, d'autres personnes ont-elles eu une influence sur vous au début de votre carrière d'écrivain?

R. L. Le premier qu'il faut ici nommer et à qui je dois davantage, à tous les points de vue, c'est Victor Barbeau. Nos échanges commencèrent par un duel, bien inégal mais qui finit très bien pour moi, mon redoutable adversaire me laissant les avantages de cet engagement.

Mon frère fréquentait alors les Hautes Études commerciales et monsieur Barbeau $y$ enseignait le français. J'insiste donc auprès de mon frère, qui redoute une rebuffade pour nous deux, pour qu'il remette mon manuscrit à son professeur. Ce qui fut fait, non sans tremblement. Le manuscrit revient avec un mot parfaitement banal. Au lieu de me résigner, je laisse monter mon indignation et je trempe ma plume dans l'encre rouge pour rappeler monsieur Barbeau à son devoir de critique et de président de la Société des écrivains. Vous croyez que Victor Barbeau s'est indigné à son tour? Non, il s'est amusé de ma naïveté audacieuse, de ma foi en lui dépassant les bornes du respect, et s'est bravement attelé au pensum de mon texte. II fit plus, dès que le texte eut paru, il le commenta à Radio-Canada, m'ouvrant pour la première fois les portes battantes de l'attention publique.

Je dois donc à monsieur Barbeau plus que le déblocage des mes débuts, mais encore un soutien sérieux, parfois et heureusement sévère, soutien qui se prolongea en amitié et fidélité. Quant à moi, je sais ce que je lui dois d'admiration et de gratitude...

J. B. Au début, vous avez donc publié vos œuvres à frais d'auteur?

R. L. II le fallait bien... comme cela se pratique encore. Mes trois premières cuuvres ont été publiées grâce à des emprunts à la banque... Les minces droits ont quand même suffi au remboursement.

J. B. Si nous en profitions pour parler de l'autre Barbeau. Marius; vous avez collaboré, je pense, pour les Madones canadiennes?

R. L. Ici encore, il s'agit d'un défi que je relevai avec joie. Marius Barbeau ayant pris connaissance de mes poèmes m'écrivit pour me demander si j'accepterais de relever par un poème quelques madones sculptées du Québec. 
Je répondis que non seulement j'aimerais écrire dix poèmes à la Vierge, mais peut-être tout un chapelet... donc 53 poèmes. Enthousiasmé, Marius Barbeau m'invita, en compagnie de sa fille et parfois de madame Barbeau, à une tournée dans la région de Québec afin que je puisse voir sur place les plus intéressantes de nos Madones. Nous visitions les couvents, les églises, les presbytères, et même les cimetières pour admirer ces Madones disséminées un peu partout dans la province.

C'est monsieur Barbeau qui décida la maison Beauchemin à publier les Madones canadiennes, édition de luxe avec lettrines en couleur, et une superbe couverture; le tout dessiné par Grace Melvin de Vancouver.

R. G. Comment avez-vous connu Alphonse Piché de Trois-Rivières?

R: L. Ce fut à l'occasion d'une conférence sur les Madones canadiennes, donnée aux Trois-Rivières. Alphonse Píché s'y trouvait avec sa sceur Aline, professeur de ballet et peintre. Après la causerie, quelques intimes dont les Piché, furent invités chez la romancière Adrienne Choquette. Se joignait à nous, l'écrivain Clément Marchand. C'est au Bien public, grâce à ces rencontres d'un soir, que j'allais publier Escales et surtout conserver ces amitiés trifluviennes.

Je relève un petit incident qui met en relief la camaraderie et la liberté de nos relations d'éditeur à auteur. Lorsque me parvinrent les premiers exemplaires d'Escales, je constatai avec stupeur que le papier comportait deux teintes de blanc et la variété commençait à peu près au milieu du volume. Je demande la raison de ce sans-gêne ou de cette fantaisie à Clément Marchand qui me répond, sans broncher ni rougir : «Écoutez, Rina, vous m'aviez dit de ménager, car vous étiez responsable de vos frais... c'est ce que j'ai fait en utilisant des restes. " Je me mis à rire... et lui aussi... Va, pour une escale à deux temps et changeante comme la neige...

\section{J. B. Vous avez aussi connu Alfred DesRochers?}

R. L. On n'oublie jamais DesRochers... Lors de cette première rencontre, nous nous trouvions chez madame Gaudet-Smet pour toute la fin de semaine. Un grand feu de bois réchauffait les conversations et bientôt ce fut entre Alfred et moi une vive discussion sur la poésie. II me reprochait le vers libre et je l'interrogeais sur les chevilles nécessaires à la poésie classique. Je constatai alors que DesRochers connaissait non seulement la poésie française, mais aussi l'anglaise et l'américaine. Que sa culture ne se bornait pas à la poésie. Ce fut une rencontre marquante, extraordinaire. D'autant plus, que dans la conversation plus générale et familière, DesRochers se taisait volontiers, comme soudain absent ou intimidé par trop de gens mis en présence les uns des autres...

R. G. Cette rencontre se répéta-t-elle? 
R. L. Oui, cette fois, accompagnés de Marie LeFranc, d'Alphonse Piché et du Père Lamarche, nous nous rendîmes chez DesRochers à Sherbrooke. Comme madame Lefranc l'interrogeait surtout sur le folklore, notre ami, Alfred, assis confortablement dans un fauteuil de barbier, se mit à chanter d'une façon extraordinaire, en scandant et tapant du pied. II chanta ainsi pendant une heure, nous racontant que la plupart de ces chansons lui venaient de sa mère... Marie LeFranc demanda à notre poète pourquoi il ne profitait pas de son immense talent de conteur et de chanteur pour gagner sa vie au lieu de piocher dans un journal de Sherbrooke?

Alfred DesRochers s'éclata de rire... et ce fut là sa réponse de poète...

\section{J. B. Et Anne Hébert?}

R. L. Je l'ai si peu connue, car que valent ces rencontres formalistes de lancement ou d'obligations sociales pour vraiment connaître une femme que l'on est déjà habitué à voir et à comprendre dans ses cuvres?

R. G. Et Grandbois?

R. L. J'eus l'occasion de le mieux connaître et de le rencontrer plus souvent. Parfait gentilhomme, fidèle ami, si ouvert et si mystérieux. Je me souviens d'une visite charmante alors qu'il habitait à Val-David, pas loin de chez Valdombre à qui nous venions de rendre visite... Après l'Ours grondant... le navigateur des astres et des fleuves nocturnes.

J. B. Et Gabrielle Roy?

R. L. Je ne l'ai rencontrée qu'une fois... Là encore, ses œuvres restent une présence, l'autre présence.

J. B. Que pensez-vous de Saint-Denys Garneau?

R. L. Je l'ai beaucoup lu au début. II nous apportait un cœur lourd dans une parole aérienne, presque... évanescente. Une jeunesse penchée, douloureuse et trop tôt fauchée...

R. G. Je suis persuadé que vous n'avez jamais lu Loranger?

R. L. Ni vu ni connu... la poésie du Québec restait toujours à découvrir, à être ressuscitée...

J. B. Gustave Lamarche?

R. L. Je l'ai connu très tard. II avait peu écrit en poésie, adonné au théâtre et donné à sa revue les Carnets viatoriens. C'est par une offre de collaboration à cette revue que j'ai mieux connu le Père Larmarche, collaboration que j'expédiais, assez régulièrement, de Saint-Jean.

J. B. Et comment Gustave Lamarche avait-il appris votre existence? 
R. L. Par l'un de ses anciens élèves qui lui avait donné à lire l'un de mes poèmes paru dans le Richelieu. Le Père, terminant sa lecture dit à son ami : "Ce doit être une femme de soixante ans pour dire ainsi les choses avec sagesse. " L'ami sourit et répondit : “Elle n'a pas vingt-cinq ans."

J. B. Aimez-vous la poésie de Gustave Lamarche?

R. L. Je l'ai dit publiquement, dans une conférence. C'est un grand poète, à la fois baroque et classique, tantôt symboliste, tantôt parnassien, mais toujours lui-même; rempli de souffle, de force, de fantaisie, et par-dessus tout, et retenez-le, un poète qui n'a jamais cessé de fréquenter son âme.

Si ses poèmes profanes étaient signés d'un nom plus profane, disons, ou d'un nom étranger, il serait au premier rang de notre poésie.

J. B. Quand même, la rhétorique dans tout cela?

R. L. Écho d'une formation classique et goût en lui du géomètre, du dessinateur-enlumineur; la ligne répondant à la ligne, la courbe à la courbe. C'est encore moins difficile à traverser que le Péguy des interminables poèmes piétineurs et qui pourtant satisfont plus d'un admirateur.

J. B. Lui reconnaissez-vous une influence sur votre poésie?

R. L. Ni dans la forme ni dans le fond. Nous sommes probablement aux antipodes. Ma forme concise, elliptique, violentant parfois le sens ne ressemble en rien aux sonnets, aux longs poèmes (tous en parfaits alexandrins) du Père Lamarche. D'ailleurs, entre poètes, les influences ne peuvent pas durer plus que l'espace d'un matin... celui des dix premiers poèmes. On dit que deux lions ne vont pas boire à la même eau; deux poètes en possession de leur don ne redoutent ni les influences ni les modes.

R. G. Bien sûr, il ne s'agit pas de la thématique biblique que l'on retrouve et chez vous et chez Gustave Lamarche, mais bien de l'écriture poétique?

R. L. Je vous l'ai dit, l'écriture poétique reste si liée à la thématique comme l'écorce à l'arbre qu'il faudrait écorcher une poésie pour n'en rejeter qu'une partie... Êtes-vous sûr de ne pas rejeter le spirituel avec le poétique?

R. G. Comme vous, sans doute, le baroquisme me déroute?

R. L. Moi aussi, mais pas au point d'obnubiler cette voix si près du cœur et de l'âme, si parfaitement libre de toute incitation à la poésie formelle, intellectuelle et plus superficielle qu'audacieuse, plus impuissante que nouvelle.

\section{Les grandes étapes de l'oeuvre : vers la maturation}

R. G. On sollicite beaucoup votre collaboration dans les revues? Cela d'une façon régulière? 
R. L. N'oubliez pas la proverbiale paresse des nôtres lorsqu'il s'agit de l'écriture, de sorte que si vous acceptez des collaborations ici et là, les demandes affluent. Ce n'est donc pas uniquement une question de qualité, mais aussi de pénurie d'écrivants.

R. G. Ces collaborations pouvaient-elles entraver votre cuvre?

R. L. Cela dépend de deux choses : de la fréquence et de la liberté accordée aux collaborateurs. Si on vous donne carte blanche, vous restez maître de votre écriture, de vos sujets. Cependant, j'ai toujours la sensation, même lorsqu'on m'accorde toute latitude, d'avoir sacrifié quelque chose à la farouche pureté de n'écrire que par l'acte même d'écrire.

R. G. Et les anthologies?

R. L. Ah! ces chapelles du chemin dans les voies de la littérature! II y a plus d'anthologistes enthousiastes que d'anthologistes sérieux. On bâtit un livre sur le dos des collaborateurs et il arrive qu'après avoir cédé ses droits d'auteur on ne reçoive même pas un exemplaire justificatif. Qu'importe, il vaut mieux que la poésie soit diffusée, même par des colporteurs distraits que laissée à ses oubliettes.

Je viens de collaborer à une anthologie européenne de mille pages; et qui donc aura le courage de ronger cette masse, de consulter ce palmarès de noms à retenir (ceux de ses amis) et à oublier...

J. B. Avez-vous beaucoup de demandes d'interviews, de rencontres?

R. L. Pas en grand nombre (nous ne sommes pas des vedettes) mais d'une façon régulière et ordinairement intéressante, je veux dire avec des interlocuteurs sérieux.

J. B. Êtes-vous sollicitée par la radio, la télévision?

R. L. Par exception, à l'occasion des prix reçus surtout.

J. B. Aimeriez-vous écrire pour la radio ou la télévision?

R. L. Très franchement, non. La raison en est que je ne suis jamais à mon aise lorsqu'il faut faire vite... Je n'ai pas oublié le labeur moins satisfaisant de mes débuts en journalisme. La date de tombée appliquée sur votre cou comme un couperet... Ouf! Vive l'espace, en soi et autour de soi.

J. B. Il y a un numéro de Liberté ${ }^{2}$ qui vous a fait énormément de bien et a servi à briser le fameux carcan mystique.

R. G. Dans l'introduction à vos CEuvres, en 1972, vous faites allusion à toutes ces incompréhensions.

R. L. Ce numéro, en effet, fut un bienfait pour mon œuvre. Par la variété des thèmes, la qualité des collaborateurs, leur bonne foi, j'ai senti qu'enfin la grande barrière des préjugés et des indifférences faciles était franchie. 
Songez à l'idéologie actuelle de la critique, de la philosophie enseignée aux universités, au mouvement des écritures tenant autant de la science que de la littérature, que pouvais-je représenter avec au fond de moi cette foi vivace partout conspuée ou répudiée?

Ayant échappé aux influences marxistes ou sartriennes, je devais faire figure d'attardée malgré la modernité de ma poésie dans sa forme et ses thèmes. D'abord bien accueillie, entre 1945 et 1955 , je sentis que le vent tournait, que la marée reculait. Et pourtant, je ne changeai rien à ma manière de penser, vivant de mes propres fonds pour rester authentique.

J. B. Comment caractériseriez-vous cette crise des années d'évolution violente?

R. L. En laissant la parole à Pierre Nepveu qui nous prévenait et nous éclairait en affirmant: «Les jeunes poètes n'écrivent plus de poèmes, mais donnent des textes dont le contenu est ou bien une préoccupation linguistique formelle, ou bien un verbalisme vide. Et cela non par une réévaluation du langage, mais par impuissance."

Vous le constatez, la crise n'est donc pas seulement esthétique mais religieuse. On veut se vider des tabous que sont les mots Dieu, mysticisme, absolu. Mot et sens remplacés par néantisation, incohérence, vérisme, désordre, sexe, vulgarité, évidence de la laideur, admiration du démentiel...

R. G. L'interprétation de votre poésie a dû souvent vous étonner ou même vous désespérer?

R. L. Je cite un cas typique de mauvaise foi. Une étudiante arrivée à son doctorat et venue me consulter au sujet de sa thèse dont j'étais le sujet... torture... releva mes remarques, mes rectifications nécessaires à des contresens, par cette parole révélatrice: “ $\mathrm{Ce}$ n'est pas là, dit-elle, l'opinion de notre directeur de thèse..." Je répondis : "Madame, êtesvous moins intéressée à la vérité qu'à l'obtention de votre doctorat? En ce cas, je n'ai plus rien à vous dire..."

J. B. Cela peut n'être que de la bêtise, mais il y a plus profond, il y a les défigures et les masques...

R. L. Le masque le plus tenace comporte deux faces fausses : la sérénité et le mysticisme. Avec ces deux mots, on pouvait expédier tout le reste. On séparait donc ma poésie de sa ferveur, de sa passion, pourtant partout évidente, mais contenue par cette concision qui fait honneur et au lecteur et à la pudeur... L'animal cache ses amours, et l'oiseau cache sa mort deux fois, et sous l'aile et sous la feuille.

J. B. J'avouerai qu'il arrive aux professeurs de se contenter des opinions courantes... de répéter les erreurs des autres. Et c'est de cette paresse néfaste que la revue Liberté aurait, du moins en partie, délivré votre œuvre? 
R. L. Justement, et fort heureusement. Là a été mieux saisi, explicité ce mélange du charnel et du spirituel qu'on n'a jamais reproché à Claudel, à Jouve, ni encore moins aux mystiques et poètes soufis.

Pour moi cet emmêlement du corps et de l'âme, du cœur et de l'esprit appartient à tout être normal qui ne s'est pas renié en cessant de fréquenter la partie la plus haute de lui-même, son âme.

Cette double part, accordée ou discordante, ne peut signifier ni distorsion ni division, mais plénitude, douloureuse ou bienheureuse.

J. B. Et si nous allions plus au centre du dynamisme de l'inspiration?

R. L. Je dirais que c'est toujours une sorte de béatitude sans facilité de plonger en poésie. J'ai l'impression d'y habiter comme en un lieu plénier, une sorte de point central d'équilibre, de solitude riche, de connaissance chaude.

Lieu de grâce aussi entre la fascination du dire et l'incarnation de la parole vivace.

J. B. Quelles ont été les grandes étapes de votre maturation personnelle?

R. L. Celles du labyrinthe imprévisible de la vie... Notre croissance suit les méandres de nos rivières intérieures comme de nos espaces aériens du désir... La poésie ne dépend ni des humeurs, ni des événements, ni des paysages, ni des moments privilégiés; elle dépend du don de poésie. C'est si vrai que le choc, la violence, la drogue ne favorisent pas la poésie.

R. G. Y eut-il des dates-charnières dans cette poursuite de votre œuvre?

R. L. Pas plus qu'on ne grandit par à coups, pas plus qu'on ne se rend compte des changements d'orientation d'une œuvre. Bien sûr, il y a des relais, des influences, des approfondissements. Ainsi, les voyages et les absences m'ont toujours été très bénéfiques. Les amitiés m'ont toujours soutenue, retenue et stimulée sans que j'en calcule jamais la portée.

J. B. Je ferais une différence entre Escales (1950) qui relève d'une thématique aérienne et religieuse et l'Arbre blanc (1966) qui développe une symbolique plus terrestre. Il y a un changement très marqué d'un livre à l'autre, ne croyez-vous pas?

R. L. Sans doute, voyez l'écart des années... Dans Escales, on voit mieux la danseuse et les impossibles envols de la faim et de la soif encore jeunes. L'Arbre blanc s'en va pensif, lucide de songe et de blessures... Le songe a la mue de la neige... monceau de larmes secrètes allant vers le printemps de l'arbre vert...

J. B. L'Échelle des anges (1975) accuse-t-il un recul?

R. L. Pour certains lecteurs sceptiques, peut-être. Pas pour moi qui ai toujours aimé et interrogé les anges. 
J. B. C'est écrit dans une prose magnifique. II y a aussi les Signes (1976), qui vient d'une écriture stricte, un peu valéryenne. Je vous avoue que j'aime énormément ces deux livres récents.

R. L. Aimer l'Échelle des anges, c'est rallonger ma joie. Les fonds de cet essai sont strictement théologiques. Le reste, tant pis pour les anges et pour moi, vient de la méditation et de l'invention du poète.

Remarquez que je ne néglige pas, pour mon usage personnel, le côté pratique de la présence de l'ange. Je dois cet usage audacieux à la suggestion d'un vieux prêtre de Lyon, comme moi adonné aux anges, non à l'angélisme.

II me conseillait de me servir de l'ange par exemple pour obtenir un taxi... Surtout l'hiver... et encore mieux, pour disposer une personne incommode ou hostile à bien écouter ce que vous avez à lui dire. Essayer le procédé... et vous vous sentirez moins seul. Remarquez que j'ai toujours voyagé seule, dans des situations parfois risquées, périlleuses, et rien de fâcheux n'est survenu.

J. B. Je suis curieux, j'insiste. Comment pouvez-vous relier la justice à la main droite du Christ?

R. L. Par l'imaginaire ou la suggestion de l'ange... et l'ange a plus d'humour et d'amour que vous n'imaginez.

J. B. Je ne vois que les Orthodoxes, dans le christianisme actuel, pour parler des anges et du Christ comme vous le faites.

R. L. Que vous me faites joie! Depuis Athénagoras et depuis les /cônes d'Evdokimov, comme de la liturgie orthodoxe, qui ne prendrait goût à ces traditions de beauté plus sensible, de sainteté jamais janséniste? Si vous avez lu l'Amour sans limites, par un moine anonyme, vous commencerez à regretter que notre formation spirituelle ait tellement manqué à cet Amour de proximité. Ainsi, saint François de Sales, suave et fort, entrerait bien dans cette lignée des mystiques orientaux.

\section{La critique de la critique}

J. B. Comment expliquer ce regain d'intérêt pour votre œuvre?

R. L. Cette explication doit vous être plus facile qu'à moi qui n'ai guère le temps d'analyser les coups de marée haute et de marée basse. II y a peut-être les prix, les honneurs, et surtout les nouveaux et plus jeunes professeurs des universités, moins prévenus que ceux de la décennie postérieure.

L'obstacle de la religiosité étant levé, les frileux ont moins honte de mon infirmité catholique pratiquante... Je ne sais et je souris. On n'aime 
pas ce qui est trop voyant et je n'ai rien dissimulé... ni retranché de ma foi heureuse.

R. G. Ce fond spirituel existait également chez Anne Hébert?

R. L. Et même chez Grandbois, pas du tout incroyant. Mais qu'étaitce que cette petite vérole ambiguë comparée à mon infection virulente?

J.B. Parmi les critiques plus compréhensifs, moins réticents, quels noms retenez-vous?

R. L. D'abord les noms de trois religieux, forcément... Hilaire de la Pérade, capucin, Gustave Lamarche, et Clément Locqwell. Puis Gilles Marcotte, avec des points d'orgue... et assez de fidélité.

Enfin, Jean Éthier-Blais, louvoyant entre l'admiration et le dédain, mais soudain superbe dans ses éclairages et ses trouvailles.

J. B. Ce sont là les critiques de l'autre génération?

R. L. Non les moindres. La jeune critique a encore des cruautés, mais semble faire effort pour, à travers ses réticences, rester objective. Songez, encore une fois, à cette collaboration de Liberté: l'essentiel des anciennes barrières a été enlevé, l'ensemble reste très positif.

De plus, les études sur mon cuvre se multiplient et à la fin, je crois que tout sera rétabli. Cependant, je relève encore et malgré tous les avertissements, des contresens parfaits chez les essayistes les mieux intentionnés. Comme il est difficile d'effacer une défigure...

R. G. Presque tous parlent de votre hermétisme, qu'est-ce que vous en pensez?

R. L. On fait mal la différence entre hermétisme et le mystère d'une œuvre. L'hermétisme vient d'une complexité impuissante ou artificielle. Le mystère, d'une ascèse de la poésie, de sa rigueur, de ses silences suspendus... II faut que l'effusion de la poésie aille vers la profondeur et ne se perde pas en jeux de clignotements.

Pourquoi voudrait-on tout comprendre alors que l'on ne se comprend pas soi-même et que l'on ignore presque tout de l'anatomie de son corps et de ses mouvements les plus simples? Et pourtant l'homme communique... et pourtant la poésie ouvre à la connaissance et à l'émoi.

J.B. Permettez-moi de forcer en quelque sorte le mystère de votre poésie. Croyez-vous que tout ce qui est privilégié, et la poésie l'est, soit difficile?

R. L. En un sens, oui. Cependant, certains poètes offrent une surface plus éclairée que d'autres, mais les fonds demeurent ténébreux par la 
richesse encore informe et insaisie et l'unité profonde du poème aveugle sur le prix de cette unité.

Je sais que la plupart de mes vers se juxtaposent plus qu'ils ne s'ordonnent et c'est perdre son temps que de chercher le fil commode du rationnel. II faut, pour goûter la poésie, renoncer aux aises et habitudes de l'esprit, établir en soi le silence et se laisser entraîner au sens profond et à l'harmonie interne du poème. Si le soleil récapitule la lumière, les reflets purs, la disposition d'accueil récapitulera le sens et le silence du poème.

J. B. Qu'est-ce que cette disposition d'accueil?

R. L. C'est une certaine capacité de recevoir la poésie un peu comme le poète la reçoit et comme en écho. C'est la capacité de voir les signes et de les suivre... de les accompagner. II s'agit donc ici de rencontre... d'abandon actif à une parole autre.

\section{Le rayonnement de la poésie}

J. B. Est-il nécessaire d'avoir une grande passion, une grande Cause, une grande vision pour être un poète qui dure? Et n'être pas seulement un artisan du langage et des mots?

R. L. Éliminons d'abord la grande Cause. La poésie est un acte, et c'est là son engagement unique. Servir et manifester la poésie sans idée de service et de manifestation.

Le seul mérite du poète, c'est de reconnaître son don, de s'en servir en l'enrichissant sans fin. Sa passion prend les deux sens d'amour et de couleur, tourment et de savoir, mais un savoir sans paix et qui demande toujours à passer par le frémissement.

L'autre savoir, celui du langage et des mots, complète celui de l'intelligence et du cœur confondus.

Octavio Paz a dit, avec humour et vérité : «La poésie est une passion, la prose, une occupation."

La vision, c'est d'adhérer à l'invisible... à l'au-delà des choses et des êtres, au plus de la vie, à cette frange de beauté que nous tend la Vérité.

J. B. II faut donc vivre sans cesse en alerte?

R. L. Oui, car tout nous fait signe, tout nous invite, de la racine au fruit, du sable à l'étoile, à la connaissance savoureuse et surréelle. La science nous y aide si nous lui restituons son âme... une âme admirative qui n'empêche pas la rigueur de la recherche. 
Nous nous servons de tout avec des mains aveugles et c'est l'aveugle lui-même qui nous apprendra à contourner la nuit en la palpant. Tout est toujours à redécouvrir, à interroger, mais avec amour.

J.B. Le mot aussi, vous l'interrogez?

R. L. Inlassablement. On ne se sert pas de mots en poésie, on sert le langage par le mot mis en rapports nouveaux. Ces mots sédimentaires, perles devenues sable, usés par la routine du vocabulaire quotidien, il faut les décaper, retrouver leur poids doré et leur ombre de silence...

J. B. D'où vient l'intellectualité d'une certaine poésie actuelle?

R. L. C'est une poésie qui a refusé son âme, surtout l'émotion qui contient le chant, et aussi cette ferveur qui favorise la communication et la communion.

R. G. Que faudrait-il changer?

R. L. La conscience... du poète. Cet effritement de la conscience était déjà commencé depuis Lautréamont, les surréalistes et les automatistes.

R. G. L'automatisme n'a-t-il rien apporté ?

R. L. Jusqu'à un certain point, il a stimulé l'imagination verbale; mais devenu procédé, il risque l'appauvrissement et l'échec. Ainsi en estil de toute poésie fabricatrice,' car la poésie ne fait pas, elle dit.

J. B. Quelle différence faites-vous entre les deux expressions?

R. L. La poésie fabricatrice se satisfait de sensations brutes, de rythmes, du jazz des mots, du tempo forcené ou désespéré du jazz. C'est une poésie matérialiste, couleur de notre époque.

J. B. On ne remplace donc jamais ce que vous appelez l'âme de la poésie?

R. L. Je ne le crois pas. Si nous nous tournons vers les païens, retenons au moins qu'ils respectaient le sacré. Ne pas respecter le sacré, c'est vite tomber dans la vulgarité et la dureté. Ici, il n'est plus question de musique ni de rayonnement de la poésie...

J. B. Actuellement, il y a une explosion du corps et de la sexualité dans notre civilisation. Évidemment, il y a état de crise.

R. L. Crise religieuse puisque même la mystique naturelle, qui n'est que le sens du dépassement et de l'invisible, s'amenuise.

J. B. Vous avez complété cette mystique naturelle par l'expérience de la foi?

R. L. Parfaitement. Tous les artistes restent des mystiques naturels, doivent se servir de leur sensualité, de leur éros pour créer. La mystique surnaturelle n'est pas une amputation, mais un achèvement. 
Elle n'émonde pas, elle surélève et enracine à la fois. Reverdy nous le rappelle: "Cette effusion de l'âme par la poésie, c'est la part de Dieu."

\section{La question féminine}

J. B. Le mouvement féministe actuel semble rejeter le dévouement, la générosité, la tendresse. Que vous en semble?

R. L. Puisque la femme a toujours incarné l'amour, malgré les abus de ce mot et de cette chose, c'est encore elle qui en suscite la merveille et en préserve le mystère. L'amour ce n'est pas surtout aimer et être aimé, c'est l'autre, c'est celui-là ou celle-là qui devient un autre moimême non pour moi-même, mais pour la joie des deux en un. Cela ne s'établit pas en peu de temps; il y faut la fidélité, la tendre amitié qui a tant souci de l'autre, et par-dessus tout, une parfaite liberté dans ces échanges qui approfondissent et élargissent l'intériorité et l'intériorisation de l'amour. Un tel amour suscite toujours un accroissement personnel remarquable. Après cela, avec cela, on ne discute plus de tendresse, de dévouement, de générosité. Tout est gagné et donné à l'avance pour les deux.

J. B. Oubliez-vous les tristesses de l'habitude?

R. L. Encore une fois, je me permets de citer Abel Bernard qui a bien écrit de l'amour: “Ce n'est plus l'Habitude qui met à l'amour une robe grise; c'est l'amour qui donne à l'Habitude une robe d'or. »

J.B. Vous sentez-vous concernée par la prise de parole des femmes, depuis dix ans, depuis Simone de Beauvoir?

R. L. Très peu, mais ce peut être un désengagement facile puisque je ne suis pas dans la lutte pour le pain quotidien, ni dans la course pour la libération de la femme, ne me sentant pas empêchée de vivre ce qui pour moi représente l'essentiel.

J. B. Et le divorce?

R. L. Ne sommes-nous pas en train d'entrer dans d'autres préjugés plus néfastes à l'amour que l'indissolubilité du mariage? Penser au divorce, et l'on y pense avant de se marier, n'est-ce pas dès le début se priver d'espérance, de stabilité? N'est-ce pas poser la souillure de l'infidélité, du féroce égoïsme avant même qu'ils ne rompent la confiance?

R. G. En littérature, mettez-vous à part l'écrivain féminin?

R. L. Pourquoi le ferai-je alors qu'il y a des hommes qui écrivent d'une façon fragile et féminine, et des femmes qui ont la force et l'envergure masculine... je songe en ce moment à Marguerite Yourcenar. 
Et pour la fragilité et la faiblesse, je songe à Paul Géraldy, à Lamartine, et à la plupart des "chansonniers" ou paroliers plus sirupeux que nos érables de mars...

R. G. Admettez-vous la complémentarité dans le couple?

R. L. Bien sûr puisque c'est là le gage de l'unité, des influences réciproques et heureuses. En amour, l'homme va vers son plaisir et la femme vers l'unité. L'homme est donc rassasiement et la femme, paix et repos. N'est-ce pas une complémentarité fondamentale?

\section{La nature et le silence}

R. G. Rina Lasnier, d'où venez-vous, quel était le paysage de votre enfance et de votre adolescence...?

R. L. L'eau, l'eau qui marche, et saute parfois «rapidieuse "... l'eau du Richelieu passant des îles de Sorel au grand lac Champlain. De la maison je pouvais voir miroiter cette rivière, et tout l'été je m'y baignais et j'y canotais.

R. G. Et vous y rêviez?

R. L. Pour réfléchir et rêver, j'empruntais plutôt le chemin de halage entre la rivière et le canal. A cette époque, on n'avait pas encore dénudé ces berges ombreuses et c'est sous de très vieux saules que je faisais halte, soit pour noter un poème, soit pour m'allonger dans l'herbe ou même grimper dans mon saule à racines rouges comme des antennes de homard.

R. G. On ne vous dérangeait pas?

R. L. Pas du tout, car c'était là la promenade des seuls pêcheurs que je saluais d'un sourire ou d'une parole. Nous nous sentions amis et liés par l'amour de la rivière et le calme de ces heures d'eau. Si bien qu'un jour, l'un des pêcheurs me proposa de me fabriquer un petit banc pour remplacer le gros coussin que je traînais dans mon sac. Je me demande encore si ce ne sont pas eux les vrais poètes du Richelieu, jamais las de ses trois saisons d'intimité, de ses anguilles aux reins puissants, de ses goélands assis sur les pierres écartées de la berge?

R. G. Vos longues vacances ont dû vous conduire vers d'autres eaux et d'autres rives?

R. L. Quand on a vu une fois la mer, on ne s'en détourne plus jamais. Aussi, j'ai passé plusieurs années de juillet à Martha's Vineyard, cette île encore préservée des inondations touristiques et des profanations radiophoniques. La plage longue et sinueuse favorise l'isolement et le silence; entre deux immensités, ciel bleu et mer verte, on réapprend le besoin de l'esprit et de l'âme: vivre l'indéfini, et l'harmonie possible, avec une forme d'absolu visible et tangible. 
Qu'est-ce qu'une piscine après cela sinon une boîte de conserve pour les étouffés des villes. Un pauvre qui barbotte, pieds nus dans un ruisseau reste plus près de l'eau qu'un riche qui nage dans sa piscine chlorurée et enserrante.

R. G. De quoi est fait votre silence, à l'extérieur et à l'intérieur?

R. L. Pour l'extérieur, nature et visages, attention, admiration, émerveillement sans lassitude, enrichissement comme par osmose et mise en branle de l'imaginaire.

Pour l'intérieur, lent mûrissement de la pensée, de la connaissance parfois comme donnée, contemplation naturelle ou spirituelle; ces approches par le silence inclinent l'esprit et l'âme comme à même l'espace de la transcendance, ainsi la branche sous la venue brusque de l'oiseau ou avec le soulèvement du vent... Le monde change... se déplace.

J.B. Regardez-vous la télévision?

R. L. Réponse facile, je n'ai pas encore de téléviseur...

R. G. Et la radio?

R. L. Je m'en sers assez fréquemment pour les émissions de musique et de littérature. J'ai aussi des disques que j'aime réentendre.

R. G. Les journaux, les revues?

R. L. Un minimum, pour rester reliée au monde et suivre les différents mouvements d'art, de littérature et de religion.

J. B. Vous ne vous laissez donc pas envahir par les mauvaises nouvelles, les désastres quotidiens, les idéologies changeantes?

R. L. Non, car il deviendrait difficile d'écrire; la poésie paraîtrait précaire devant la bombe atomique ou la torture; et pourtant, il n'y aura ni paix, ni justice sans beauté, sans poésie.

J. B. Que pensez-vous des poètes engagés?

R. L. Leurs raisons ne manquent pas de noblesse et de générosité, mais le risque est grand que la poésie s'évapore dans la chaleur ou l'artifice de l'engagement...

R. G. II existe plusieurs sortes d'engagement...

R. L. Certes et Roger Bastide ne sera pas le dernier à s'être laissé prendre au piège de sa propre renommée et à avoir accepté un poste à la radio-télévision italienne. Depuis ce changement, depuis dix ans, il n'a rien écrit...

J. B. Avez-vous à refuser beaucoup d'invitations? 
R. L. Cela dépend. De la part de la radio-télévision, non; mais de la part des sociétés de toutes sortes, oui. Et je me vois maintenant obligée de mettre un frein à l'aide aux jeunes poètes, faute de temps...

J. B. Mais vous vivez seule, ici, dans cette grande maison...

R. L. Justement, je fais tout et je vois à tout comme économe, ménagère, hôtesse, secrétaire de mes propres obligations de copie et de correspondance. II faut beaucoup de temps pour coordonner tout cela sans négliger les sorties et les divertisséments nécessaires.

J. B. On vous dérange beaucoup?

A. L. Constamment, mais bien involontairement. II y a une façon de se défendre sans blesser personne et de préserver son intimité. Les écrivains à domicile sont un peu comme les anges... on ne les voit jamais comme écrivants mais comme personne seule et disposant de beaucoup de temps. Une brave femme me demandait un jour: «C'est-y quand vous vous ennuyez que vous vous renfermez dans vos papiers?"

\section{Le Journal}

J. B. Et vous songez à n'écrire que de la poésie?

R. L. Pas exactement puisque j'ai à terminer un manuscrit de proses spirituelles. Cependant, il est sûr que la poésie gardera toujours la première place.

J. B. Vous ne travaillez pas à l'intérieur de projets?

R. L. Rarement. L'aventure, l'inédit, c'est de s'asseoir chaque matin devant sa table de travail et de ne pas savoir encore ce que l'on écrira. Tout le désert pour un oasis... ou une dure sécheresse, car il arrive que l'on éprouve son vide et son impuissance. A ce moment, il ne faut pas s'entêter, mais s'évader.

J. B. Avez-vous déjà accepté des commandes?

R. L. Oui, par exemple pour le Jeu de la voyagère, mettant en scène Marguerite Bourgeoys; puis pour l'album commandité par Ottawa, album contenant environ deux cents photographies et donc autant de légendes pour éclairer ces images en noir et blanc. Pour les Madones canadiennes, je vous l'ai dit, il ne s'agissait pas de commande mais bien d'un défi amical que j'ai voulu relever.

R. G. Et le roman?

R. L. Je n'en écrirai jamais... je manquerais de tout pour ce genre d'écriture, et surtout de patience. J'aurais aimé revenir au théâtre, mais j'ai constaté que là encore, il me manquait trop de formation, celle du départ, puis celle de l'expérience d'écriture. 
R. G. Les confidences ne vous intéressent pas?

R. L. Pas encore... attendons la vieillesse qui se parle à elle-même...

J. B. Il n'y a rien à tirer de votre journal?

R. L. Vous me faites soupirer... Je suis à l'avance révulsée devant les indiscrétions inévitables, et devant certaines blessures de manipulateurs de confidences et d'âmes... Si le temps de la relecture ne m'est pas laissé, tout sera détruit...

J. B. Vous ne tenez plus votre journal?

R. L. Non, j'ai cessé sans autre raison que la paresse ou peut-être une certaine plénitude de vie qui se passe de son propre secret.

R. G. Comme pour Grandbois, vous seriez horripilée si on venait, après votre disparition, publier ce que vous avez jugé impubliable?

R. L. Certes... je pense qu'en dernier ressort l'écrivain est l'ultime juge du meilleur et du pire de son œuvre. C'est bien assez des légendes souvent inventées par distraction ou besoin d'apporter du neuf ou du scandaleux. C'est bien assez d'être ligotée dans l'innocence de la mort et d'abandonner cette faiblesse à plus faible que soi...

R. G. Votre correspondance vous défendrait-elle mieux?

R. L. Des préfaciers, oui; mais la vraie défense doit être celle de l'œuvre; la biographie satisfait plus la curiosité que la sympathie.

J. B. Qui sont vos correspondants?

R. L. Des amis, des artistes, des inconnus... reconnus en poésie. Ainsi, j'ai longuement correspondu avec un poète mexicain que je n'ai jamais rencontré. Nous avons un jour découvert que nous étions nés le même jour... un jour de grande lumière, la Transfiguration.

\section{Le tourment de la création}

J. B. Je voudrais vous poser une question au sujet d'un mot que j'aime beaucoup et qui revient dans vos poèmes des Signes (p. 109). Je cite : “L'invisible nous regarde." "L'amour voit l'Amour." "Béance de la parole énergique et sèche des images». "L'esprit passe l'anneau agrandi du firmament, resserre son erre, et dans l'eau ronde du regard, franchit son infranchissable." Au point de vue de l'image, de la rhétorique, de la figure, qu'est-ce que représente pour vous l'anneau?

R. L. Peut-être la fidélité parfaite, et surtout la communication avec le Tout; car tout se trouve entre l'Alpha et l'Oméga.

J. B. Donc, une figure de la totalité? 
R. L. Oui, une totalité qui ne limite pas mais affranchit.

J. B. Et qu'est-ce qui affranchit?

R. L. L'amour... la communion qui consomme la communication.

R. G. Vous avez lu l'Arche de Grandbois?

R. L. . Oui... l'arche peut bien être aussi l'arc-en-ciel, la paix après la rupture, l'alliance après la fuite ou l'éloignement.

R. G. Cette figure de la rondeur vous est connivente?

R. L. Sans doute puisqu'elle m'habite. Marcel Bélanger l'a déjà noté dans l'un de ses articles. Songez à la perfection, pour la main, le goût et l'œil, de l'orange. Quelle plénitude savoureuse et simple, concédée à l'avance.

J.B. C'est peut-être exagéré et injuste, mais j'accorde une très grande importance à ces deux récents livres que sont les Signes et l'Échelle des anges. Je les aime vraiment et je crois qu'il y aurait là l'occasion d'un travail à effectuer sur la rhétorique et les figures.

R. L. C'est sans doute déjà commencé avec la récente étude de Sylvie Sicotte, l'Arbre dans la poésie de Rina Lasnier. J'ai bien apprécié la préface de son ouvrage sérieux et bien fait. Quelques contresens, toujours dans l'exagération mystique, mais dans l'ensemble un texte intelligent, compréhensif et très sympathique.

J. B. Vous avez lu l'étude d'Escales par Chanel Malenfant?

R. L. Certes, et encore là une étude remarquable; mais ne le lui dites pas, j'aime encore mieux ses poèmes ${ }^{3}$, je m'y retrouve mieux.

R. L. Je vous interroge à mon tour, monsieur Bonenfant; dites-moi, comme professeur, avez-vous été intéressé à la dernière partie des Signes, ces poèmes retrouvés et récurrents?

J. B. Ah oui! II faudrait que tous les écrivains en fassent autant.

R. $L$. Je voulais reprendre ces beaux déchets que laisse la première rédaction d'un poème... On croit rejeter l'ivraie et c'est l'ivraie qui refleurit. Surtout, en pleine création, tout se passe si rapidement, à de telles profondeurs nocturnes qu'on laisse filer et se noyer du beau gibier.

J. B. La substance poétique vous est donc toujours nombreuse?

R. L. C'est le subconscient qui l'est... Nos trésors enfouis ou endormis remontent sous l'étincelle de l'imaginaire, tout de suite jugé par la lucidité. Ce désordre éclairé constitue la prise et la perte du matériau brut. Ce matériau perdu peut être récupéré plus tard, à un moment imprévu et plus ou moins lointain. 
J. B. Par exemple, "J'avais un grand arbre vert", qui plus tard revient nous alerter avec ce vers nouveau: "L'arbre, flamme rugueuse de l'amour inépuisablement vert».

R. L. Vous l'aurez remarqué, j'ai aussi fait la réduction d'un texte. D'abord une prose, puis une lettre, puis le concentré du poème. L'inverse pourrait également se pratiquer.

J. B. Le recueil peut-être le plus difficile, c'est la Salle des rêves. Le poème qui donne le titre au livre me paraît très difficile.

R. L. Et pourtant, c'est celui-là que la Hongrie vient de retenir pour une traduction.

J.B. Je le trouve extraordinaire, je le comparerais au «Cimetière marin ", comme travail poétique.

R. L. Pourquoi pas à la «Malemer " puisqu'il s'agit d'eau océane?

J. B. Je suis moins sensible à la "Malemer".

R. L. Pour moi, ce poème est le tourment et la passion d'écrire en poésie. Les solitudes noires et pourtant sûres des richesses des basfonds de la mémoire et de l'incantation de l'imaginaire.

J. B. Y attachez-vous le sens de malheur?

R. L. Non pas de malheur, mais d'une sorte de mort; la mort n'est pas un malheur, mais une transformation. Ainsi la création qui nous tue et nous transforme.

R. G. Aimez-vous entendre dire vos poèmes?

R. L. Par un diseur qui ressent fortement la poésie, oui, car il se produit comme un décalage, un clivage entre le poète et ce moi qui s'entend parler une langue familière et soudain un peu étrangère. C'est à ce moment qu'importe la musique du poème.

R. G. Pour vous, la poésie est sœur de la musique?

R. L. Alain disait mieux encore quand il affirmait: "La poésie est la musique de la pensée»... car la poésie pense, contrairement à ce que certains prétendent.

R. G. La musique compte beaucoup dans votre vie?

R. L. Autant que la danse a compté dans ma jeunesse, et les spectacles de ballet aujourd'hui.

R. G. Faites-vous vous-même de la musique?

R. L. Plus maintenant. J'ai appris et le piano et le violon.

R. G. Je reviens à cette constante dont nous parlions, à savoir la crainte que vous avez d'être mal interprétée. Chaque interprète ne peut-il étudier la part de l'œuvre qui le retient davantage? 
R. L. Je ferais une différence entre l'étude et l'interprétation. II va de soi que l'analyse de telle ou telle composante de l'œuvre ne gêne guère le sens de l'ensemble.

Mais si l'analyse ou la critique prétend incliner l'œuvre dans un sens directement contraire à celui de l'auteur, il y a quelqu'un qui se trompe... Et je ne suis pas portée à croire que ce soit le poète qui ne se souvient plus de ce qu'il a voulu exprimer ou de ce qu'il est. La science ne remplace pas le discernement du créateur ni surtout la force originelle et originale de son inspiration. Le don poétique reste indivisible.

R. G. Dans son article, publié dans Liberté, Joseph Bonenfant a bien utilisé le procédé disons "centriste", greffant tout sur l'importance de l'image, et vous vous déclarez très heureuse de cette a iconographie":

R. L. Tout à fait heureuse parce que le procédé n'a été que le point d'appui du rayonnement de toute l'œuvre. Ce n'est pas en vain que dans le titre de cet article, le critique utilise le mot dimensions, et si je ne m'abuse cela signifie hauteur, profondeur, projections horizontales.

R. G. Je serais incapable d'écrire un texte comme celui de Joseph Bonenfant qui est, beaucoup plus que moi, près de votre esprit.

R. L. Vous avez la franchise de le dire et l'honnêteté de reconnaître qu'une étude écrite à chaud, comme en sympathie préalable, va ordinairement plus loin qu'une critique à froid, pleine de réticences ou comme cela est arrivé pour moi, d'hostilité presque rageuse.

J. B. Puisque vous m'impliquez si amicalement, je vous avoue que j'ai l'impression que m'eut convenu aussi une étude sur Mallarmé ou Lautréamont.

R. L. Je vous comprends de ne pouvoir ni vouloir vous borner à vos seules affinités. Reste le défi que nous proposent certaines poésies, menées aux antipodes de nos goûts personnels et de nos inclinations. II y faut plus de détachement et peut-être l'attrait ancien de l'inconnu, du périlleux, parfois du pervers. Nous sommes des créatures ambiguës et ambidextres... ramassant d'une main ce que nous brûlons de l'autre... la pomme et la nudité contre la Parole et l'Alliance.

\section{0. Écrire, lire la poésie}

J. B. Avez-vous parfois besoin de lire pour écrire?

R. L. On a toujours besoin de lire pour tout, à tous les points de vue. Quant à moi, il me faut établir une distance nette et large entre ce que je lis et ce que j'écris. La liberté ne se marchande pas, ne souffre pas les étais, les emprunts.

R. G. L'impact doit donc rester indirect? 
R. L. Vous dites bien. Par exemple, si un poème m'a enthousiasmée ou profondément touchée, j'éviterais, même si j'avais en réserve un thème qui lui était apparenté, j'éviterais de m'en servir ou alors je le ferais seulement après un long délai.

R. G. Les thèmes de vos propres poèmes vous habitent-ils longtemps avant que vous en veniez à la rédaction?

R. $L$ Cela ne se passe jamais de la même façon; certains poèmes naissent avec l'impétuosité du torrent, d'autres stagnent comme des étangs morts; on les croit ensevelis dans leur sommeil et soudain un charme les ravive.

J'ai attendu près de dix ans le poème des "Martinets". J'ignore pourquoi, mais je commence à soupçonner que ce poème qui me rappelait les dernières années de ma mère suscitait en moi trop de douleur pour que je puisse maîtriser cet ancien choc, ces images quasi funèbres des martinets noirs que ma mère regardait tournoyer, le soir... Martinets qui, le jour, se cachaient dans les grandes cheminées de notre toit, et le soir, ne cessaient de crier, de tourner dans la nuit comme des astres de deuil, cherchant une nourriture, invisible pour nous.

J. B. Dans les Signes, vous parlez aussi des oiseaux, c'est extraordinaire comme images et figures.

R. L. Les oiseaux sont des êtres privilégiés, à la fois aériens et terrestres, aquatiques et arboricoles, mais jamais souterrains. De plus, comme j'habite mon jardin et que les arbres habitent ma maison, ce sont les oiseaux qui me font le plus souvent signe, soit pour la nourriture que je leur fournis, soit pour la joie qu'ils me proposent en s'interposant entre moi et la page barbouillée ou le livre intact... ils sont comme la magnifique rature du temps perdu à les chanter... eux qu'il faut écouter.

R. G. Croyez-vous aux signes astrologiques?

R. L. Pas du tout, même si je sais que comme être vivant nous vivons tous sous une certaine influence astrale. Cette influence astrale s'adresse plus au corps qu'à l'esprit. Aussi, je me sens beaucoup plus reliée à mon ange-compagnon qu'aux planètes. L'ange est sourcier de lumière, les astres ont toujours appartenu aux sorciers et aux devins, fumeux ou dangereux.

J. B. Je vous appellerais volontiers «fille de la lumière » à cause de votre naissance et de votre œuvre tournant dans l'orbite de la Résurrection.

R. L. Ce serait là le plus précieux hommage. Vivre, ce n'est ni jouir ni souffrir ni mourir, mais savoir que nous sommes déjà des ressuscités... des vivants. 
J. B. Une idée fixe me revient, pour terminer, comment reliez-vous la poésie à l'Écriture sainte, à la spiritualité dans ce siècle de plus en plus résolument laïque?

R. L. Je ne fais que suivre la voie la plus ancienne et la plus sacrée de la poésie. Songez aux Livres sacrés des plus vieilles civitisations, aux traditions orales et religieuses des peuples à peine civilisés; songez aussi aux honneurs extraordinairement rendus, par le roi, aux bardes de l'Irlande, à l'importance des conteurs-poètes, en Afrique, Océanie, et même en Haïti de nos jours.

Ainsi, la poésie a toujours été intégrée au sacré; l'homme a toujours fait confiance à la poésie pour témoigner de son âme ou de Dieu. Tournez le grand livre de l'anthropologie et se soulèveront de la poussière les parfums vastes de la poésie transcendante...

1. Joseph Bonenfant et Richard Giguère ont rencontré Rina Lasnier le vendredi 9 décembre 1977, à son domicile de Joliette. L'entretien, enregistré sur bande magnétique, dura plus de quatre heures. Après avoir transcrit et dactylographié l'entrevue, Joseph Bonenfant et Richard Giguère ont retourné le texte à Rina Lasnier pour commentaires et corrections. Vous venez de lire le texte remanié et entièrement réécrit par Rina Lasnier. Les sous-titres sont des auteurs.

2. Numéro 108, novembre-décembre 1976.

3. Voir Poèmes de la mer pays, Montréal, Hurtubise-HMH, “Sur parole», 1976. 